\title{
Spatial working memory load affects counting but not subitizing in enumeration
}

\author{
Tomonari Shimomura • Takatsune Kumada
}

Published online: 14 June 2011

(C) Psychonomic Society, Inc. 2011

\begin{abstract}
The present study investigated whether subitizing reflects capacity limitations associated with two types of working memory tasks. Under a dual-task situation, participants performed an enumeration task in conjunction with either a spatial (Experiment 1) or a nonspatial visual (Experiment 2) working memory task. Experiment 1 showed that spatial working memory load affected the slope of a counting function but did not affect subitizing performance or subitizing range. Experiment 2 showed that nonspatial visual working memory load affected neither enumeration efficiency nor subitizing range. Furthermore, in both spatial and nonspatial memory tasks, neither subitizing efficiency nor subitizing range was affected by amount of imposed memory load. In all the experiments, working memory load failed to influence slope, subitizing range, or overall reaction time. These findings suggest that subitizing is performed without either spatial or nonspatial working memory. A possible mechanism of subitizing with independent capacity of working memory is discussed.
\end{abstract}

Keywords Subitizing $\cdot$ Counting $\cdot$ Visual working memory Dual-task performance

\footnotetext{
T. Shimomura $(\bowtie) \cdot$ T. Kumada

National Institute of Advanced Industrial Science and Technology, Central 6, 1-1-1 Higashi,

Tsukuba 305-8566, Japan

e-mail: to.shimomura@aist.go.jp

T. Kumada

e-mail: t.kumada@aist.go.jp

T. Shimomura

Chukyo University,

Nagoya, Japan

When one decides how many objects make up a scene, it is well known that reaction time (RT) for responding does not increase linearly as a function of the number of objects (Jensen, Reese, \& Reese, 1950; Jevons, 1871; Oyama, Kikuchi, \& Ichihara, 1981; Trick \& Pylyshyn, 1993, 1994). Instead, RT for enumeration typically remains roughly constant up to approximately four items in a scene; it then increases linearly with the number of to-be-enumerated items. Several lines of research indicate that this discontinuity in RT reflects the operation of two distinct psychological processes that underlie the broader phenomenon known as enumeration. For enumerating a smaller number of items, the process is rapid and accurate; this is referred to as subitizing (Kaufman, Load, Reese, \& Volkmann, 1949). In contrast, in enumerating larger numbers of items (e.g., above four), the process is timeconsuming and errorprone; this has been referred to as counting. In this study, we focus upon certain factors that determine the range of subitizing.

Previous research, which has used a variety of stimulus displays to study enumeration, offers a consensus that the number of items that can be subitized is approximately four. This holds for various displays consisting of different visual features (Frick, 1987; Watson \& Maylor, 2006; Watson, Maylor, \& Bruce, 2005). In addition, Watson, Maylor, and Bruce (Experiment 3) examined enumeration performance with a display consisting of dots differentiated by colors. They investigated the possibility that color differences might enhance individuation among dots and,thereby, affect subitizing. However, this was not observed. Instead, the range of subitizing remained constant at around four items regardless of their colors. Trick and Pylyshyn (1993) also found a constant range of four items indicative of subitizing in a task where participants were required to enumerate target items amid distractors. They used horizontal bars as targets and vertical bars as distractors; they found that the 
subitizing limit of four items was pegged to targets regardless of the presence of distractors. These results are consistent with the interpretation that subitizing reflects a distinct process with a limited capacity of four items.

Three main accounts address the process underlying subitizing and its limited range. First, Trick and Pylyshyn (1993, 1994) suggested that a subitizing range is constrained by a fixed number of mental tokens termed FINSTs (fingers of instantiations; Pylyshyn, 1989; Pylyshyn \& Storm, 1988). The FINST system provided for indexes to spatial positions up to a maximum of four simultaneously presented items. Once tokens are bound to items, FINSTs can keep track of the items even if the items move to different positions or change their appearance. The index then allows spatial attention to rapidly access items. The FINST hypothesis holds that if the number of items presented does not exceed a fixed number of mental tokens limit, the set of mental tokens can be allocated to each item without attention. In turn, this would result in rapid enumeration of items when four or fewer are present. In contrast, if the number of items exceeds the fixed number of tokens, additional mental processing is required. In this case, the tokens allocated to the initial four items have to be serially reallocated to additional items. This reallocation of tokens requires attention, following which serial enumeration occurs, and also involves attentional shift. In sum, the FINST account proposes that a limited number of tokens are available for use by the visual system at a given time and that this constraint is responsible for the subitizing range.

A second account proposes that the configuration of stimulus items determines a subitizing range (Mandler \& Shebo, 1982). According to this account, arrays with fewer items are more likely to match a familiar, canonical pattern (e.g., three dots on a dice) that is stored in long-term memory, together with other canonical configurations. In these long-term memory representations, a distinct number is also correlated with each configuration. A match with a stored configuration is likely to be quicker in displays with fewer visual items than in displays with many items, thus leading to fast retrieval of an associated numerical value. As the number of items increases, pattern matching becomes slower, due to the complexity of both presented and stored arrays; thus, retrieval of a number becomes less efficient. This account implies that the range of subitizing is not necessarily fixed; rather, it can vary depending on whether a presented configuration of stimulus items allows for an easy pattern match. But the range tends to be fairly stable. Furthermore, the assumption that canonical configurations uniquely correspond to certain numerical values is questionable. For example, although four dots are readily mapped onto a square pattern, a configuration of four dots could also represent a triangle pattern. In this case, the presented configuration should disrupt a correct decision.
Although the configuration of stimuli may contribute to the subitizing range, it is possible that this is specific to certain stimuli and to regularly presented items.

Finally, the research most relevant to the present study has been reported by Klahr (1973) and Cowan (2001). In these studies, it was proposed that subitizing range reflects limits of short-term, or working, memory. This hypothesis assumes that subitizing employs a tagging mechanism that prevents enumerating the same item more than once; limits on working memory then place constraints on how many items this tagging mechanism can track simultaneously. Therefore, working memory should allow for rapid and correct enumeration when the number of visual items does not exceed the visual working memory capacity. Furthermore, it has been suggested that the capacity of visual working memory is around four objects, which is similar to the limit of subitizing (Luck \& Vogel, 1997; Pashler, 1988; Vogel, Woodman, \& Luck, 2001). The comparability of these limits suggests that a common process may be involved in both subitizing and working memory. However, to the best of our knowledge, this possibility has not been examined empirically.

Apparently, subitizing range resembles the visual working memory span reported in recent studies. Luck and Vogel (1997) showed that visual working memory could retain up to about four objects, where the objects differed with regard to multiple features, such as shape, color, and so on. In their experiment, a participant had to retain (for $900 \mathrm{~ms}$ ) the colors of all objects presented in an initial display and then report whether or not they detected a change of color in a single object in a test display. Although the color of only one object could change for test, the number of items presented in the initial or in the test display varied from 1 to 12 . Results indicated that accuracy remained nearly perfect for 1-3 items but declined systematically for 4-12 items. Another experiment showed that accuracy of remembering both color and orientation of objects was comparable to accuracy of memory for color or orientation alone. No significant difference in accuracy was found between 2 and 4 items; however, significant accuracy differences were observed in comparing displays with 6 items with those displays containing 2 or 4 items. Taken together, such results suggest that nonspatial visual working memory has the capacity to simultaneously maintain up to about 4 items, but not more.

The present study directly investigated whether the subitizing range involves visual working memory. Given that previous studies showed that the subitizing range was comparable to that of working memory, our working hypothesis in this research was that visual working memory capacity determines the range of subitizing. This hypothesis is reasonable if we assume, as well, that enumeration of a set of discrete items requires remembering the locations of items that already have been enumerated in order to distinguish these items from those not yet enumerated. If 
remembering already enumerated locations is mediated by visual working memory, the number of items that an individual may keep track of should depend on the capacity of visual working memory - that is, its availability for enumeration. Assuming that the capacity of visual working memory is restricted to about four objects, this hypothesis predicts that subitizing will not occur beyond this limit. In other words, five or more objects cannot be simultaneously held in memory; capacity limits imply that with a large number of items, one or more will be lost, and this leads to inefficient enumeration. Thus, in this study, we examined whether a concurrent working memory load affects the range and efficiency of subitizing. If the load on working memory reduces its available capacity for enumeration, the subitizing range should decrease even when displayed items number four or fewer. This means that subitizing efficiency, which is typically indexed by the slope of an RT function over the number of items in one to three items would change accordingly (e.g., Trick, 2005; Trick \& Pylyshyn, 1993; Watson \& Maylor, 2006; Watson, Maylor, Allen, \& Bruce, 2007; Watson et al., 2005). Thus, in this case, instead of a flat slope of the RT function over one to three items, a greater memory load will elicit a positively inclining slope indicating that more processing time is required for displays even within the conventional subitizing range.

It is possible that visual working memory consists of two different subsystems. One subsystem may involve processing spatial representations of items, and the other would be dedicated to nonspatial visual representations of object features (Baddeley \& Logie, 1999; Logie, 1995). In the present study, we refer to the former subsystem as spatial working memory and the latter as nonspatial visual working memory. The present study investigated the relationship between these subsystems and subitizing.

Previous studies have suggested a relationship between enumeration and working memory. Logie and Baddeley, (1987) examined enumeration performance, as a primary task, while participants performed either articulatory suppression or tapping as a concurrent secondary task. The reasoning was that articulatory suppression and tapping should interfere with rehearsal of verbal and spatial working memory, respectively (e.g., Cocchini, Logie, Della Sala, MacPherson, \& Baddeley, 2002; Logie, 1995). Logie and Baddeley found that RTs for enumeration were longer with articulatory suppression than with either the tapping task or no secondary task. However, more important for the present purpose, the effect of articulatory suppression on enumeration was found only when the number of displayed items was large, and not when the number of items fell with the conventional range for subitizing. A recent study using either articulatory suppression or tapping as a secondary task reported a similar result, showing that performance efficiency on the primary enumeration task did not suffer from either of these secondary tasks, although RTs increased, overall, for displays with all numbers of items (Trick, 2005). These studies suggest that subitizing occurs without rehearsal of the enumerated stimuli.

A different line of research also showed that "verbal" working memory was not responsible for subitizing range. Tuholski, Engle, and Baylis (2001) and Barrouillet, Lépine, and Camos (2008) investigated whether performance of the enumeration task was affected by individual differences in working memory capacity. Working memory capacity was measured with an operation span test or a reading span test. Tuholski et al. found that a group of participants with high working memory capacity enumerated faster than those with a low capacity when the task included more than four items. By contrast, they found no difference due to capacity within the subitizing range; the subitizing range was approximately four in both capacity groups. This indicated that the range of subitizing was not constrained by verbal working memory capacity.

The goal of the present study was to explore the respective roles of spatial and nonspatial working memory in enumeration. While previous research has shown that neither a rehearsal process of working memory nor the capacity of verbal working memory is responsible for subitizing, it remains unclear whether the capacity of visual working memory plays a role in subitizing. Although previous studies have reported that neither articulatory suppression tasks nor tapping tasks interfered with subitizing, these tasks interfered only with rehearsal process and did not directly affect the capacity of visual working memory. Therefore, in the present study, we focused specifically on the impact of load on visual working memory capacities in the enumeration task. Specifically, we examined participants' performance on subitizing when visual working memory capacity was filled with concurrent visual information, using a dual-task procedure that consisted of a primary enumeration task and a secondary working memory task. In different experiments, we considered, respectively, two different working memory tasks-namely, spatial versus nonspatial tasks. If subitizing involves a specific capacity limit on working memory - that is, due to the load incurred either from a concurrent spatial or from nonspatial visual working memory-performing a working memory task (spatial or nonspatial) concurrently with an enumeration task should result in interference, evident in poorer performance on the primary task. That is, in a dual-task paradigm, if subitizing requires visual working memory capacity, performing either secondary memory task should produce a decline in the subitizing range. 
Specifically, not only should the range in subitizing be reduced, but also efficiency should be degraded as a function of the load imposed upon memory by a secondary task. Inference is predicted in these cases, due to a competition for common memory capacity. Alternatively, if subitizing is not associated with the limited capacity of working memory, performance of subitizing should be comparable to that without a working memory task. A similar logic of dual-task interference was used for combining a visual search task with a working memory task (Woodman \& Luck, 2004). Woodman and Luck found that efficiency of detecting a target among distractors in a visual search task decreased with a concurrent spatial working memory task, where efficiency was indexed by the slope of the function relating RTs to search display size. They concluded that this was due to the search process requiring participants to maintain locations of searched items. Using a similar rationale, we presented a spatial and nonspatial working memory task concurrently with an enumeration task: Experiments 1A, 1B, and 1C used a "spatial" working memory task in which participants had to remember the information about the spatial locations of objects in a working memory task, whereas Experiments 2A and 2B used a "nonspatial" working memory task in which participants had to remember the shape of objects.

\section{Experiment 1A}

Method

Participants Twenty-four undergraduate or graduate students (17 males and 7 females), 19-36 years of age ( $M=$ 22.0 years), participated for payment. All reported normal or corrected-to-normal vision.

Stimuli and apparatus Stimuli were generated using MATLAB and Psychophysics Toolbox (Brainard, 1997; Pelli, 1997). They were presented on a 17 -in. CRT monitor at a viewing distance of $57 \mathrm{~cm}$. Samples of stimuli, as they appear within a typical trial, are given in Fig. 1a. Stimuli in a display were presented on the monitor screen with a black background and a gray fixation cross at the center that remained visible throughout a trial. Enumeration stimuli were green dots $\left(0.8^{\circ} \times 0.8^{\circ}\right)$, and memory items were gray squares $\left(0.2^{\circ} \times 0.2^{\circ}\right)$. Mask items (which followed an enumeration display) were identical to enumeration stimuli. All three types of items were presented on two different spatial scales of invisible matrixes, one embedded within the other. The larger matrix was used for the enumeration array; it was composed of $7 \times 7$ cells. The central $3 \times 3$ cell region of the larger matrix was replaced with the smaller matrix scale of $6 \times 6$ cells, which was used for the memory

A

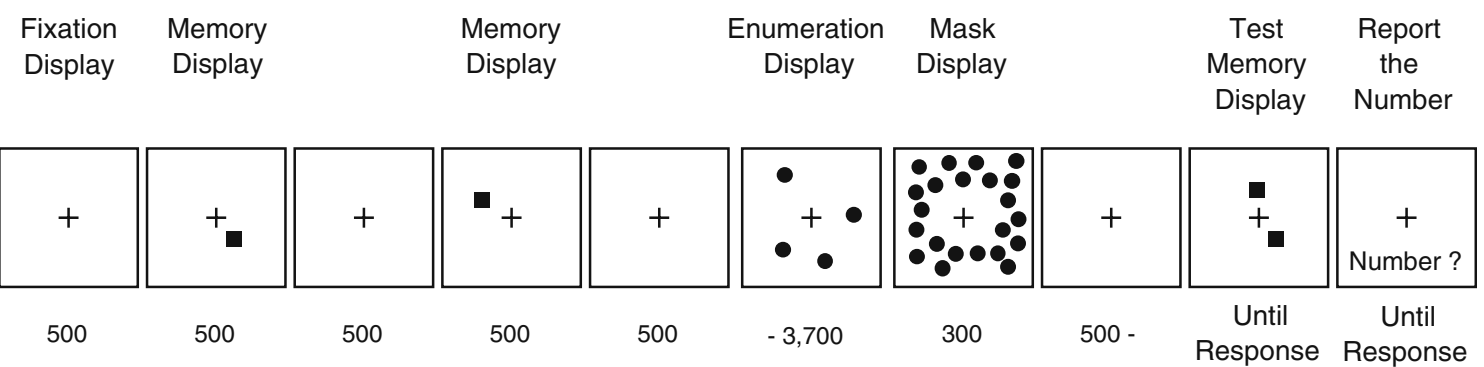

B

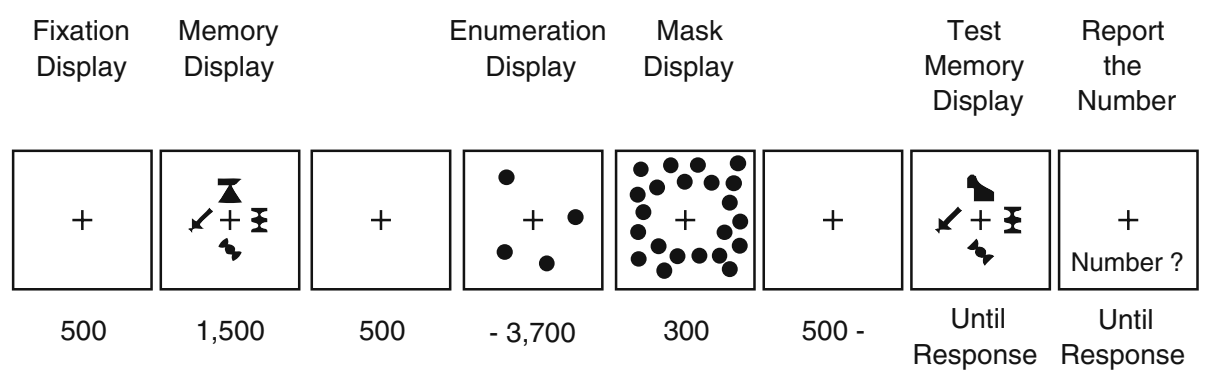

Fig. 1 An example of a trial in Experiment 1A (a) and 2A (b), respectively. The sequence was identical in both experiments, except that the memory task was remembering either position (Experiment $1 \mathrm{~A}$ ) or shape (Experiment 2A) of memory items. Presentation time of each display is shown in milliseconds. The number of enumeration

stimuli is selected from one to eight (here, four stimuliare presented). The enumeration display was present either until a response was made or $3,700 \mathrm{~ms}$ had elapsed if no response was made, which was followed by a mask display. Every task condition consists of the same sequence, but just different responses were required 
array. No items were presented in the central $2 \times 2$ cells of the smaller matrix, in order to prevent overlap with a fixation cross. Thus, the dots for enumeration and squares for memorization were always presented within spatially distinct regions. For the enumeration array, center-to-center distances of adjacent cells were $2.29^{\circ}$ horizontally and $2.48^{\circ}$ vertically. For the memory array, these were $1.37^{\circ}$ and $1.48^{\circ}$, respectively. Enumeration dots were presented randomly on the cells, with jitter up to $0.64^{\circ}$ from the center of the cell for the $x$-axis and $y$-axis. The mask array was generated using the same matrix as the enumeration array; it consisted of 40 items in all cells in which the dots could appear.

Design and procedure This experiment included a dualtask condition and two single-task control conditions. Control conditions were enumeration-alone and memoryalone conditions. The structure of a trial sequence of displays, shown in Fig. 1a, remained the same in all three conditions; only task(s) differed as function of condition. Participants performed in all three conditions. In the two control conditions, they performed only one task, whereas in the experimental, dual-task condition, they concurrently performed both a primary (enumeration) and a secondary (memory) task. In addition, the number of enumeration items varied, ranging from one to eight.

In the dual-task condition, each trial began with a 500$\mathrm{ms}$ fixation display, followed by an initial memory display, enumeration display, mask display, test memory display (interleaved blank displays had only a fixation cross), and report duration. The initial memory display comprised two sequential presentations of a square for $500 \mathrm{~ms}$, separated by a 500-ms blank screen. In the dual-task condition, a participant was required to remember the locations of both squares. These items were positioned in the smaller matrix, with the constraint that each item was never presented on cells adjacent to the cell of another item. Another 500-ms blank screen display occurred and was followed by an enumeration display consisting of one to eight dots. In the dual-task condition, a participant was then asked to press the space key as soon as he/she comprehended the number of the items. This display remained on the screen either until a participant made a response or until a total of $3,700 \mathrm{~ms}$ had elapsed from onset of the enumeration display if the participant made no response. If no response was made to the enumeration display until the display disappeared, a warning message and beep were presented at the end of the trial. The mask display then appeared for $300 \mathrm{~ms}$, after which the screen remained blank for a variable duration based on the enumeration response time to ensure a retention interval of 5,000 ms. Next, a memory test display, composed of the two squares, was presented. On half the trials, the two squares were presented at the same locations as in the initial display. On the remaining trials, one square was realigned to a new location that adjoined its original location in the matrix, with the constraint that the location was selected from the cells that were never adjacent to the other memory item. A participant made a response to indicate whether or not these test squares were at positions identical to those in the initial memory display by pressing the zero ("0") key or the period (".") key, respectively. If an incorrect response was made, a feedback beep was presented. Then the participant reported the number of enumeration items, using a numeric key. It was emphasized that the participant's initial numerical judgment of items, given by a space key press, could not be altered. No feedback was given for this enumeration response.

In the memory-alone condition, with the same displays and trial format as in the dual-task condition, participants were instructed to perform only the memory task. They had just to respond to the onset of the enumeration display by pressing the space key; they were told to do this as quickly as possible and to ignore the number of items presented. This procedure was used to ensure that overt responses to displays during the retention interval had no effect on memory performance. This condition then provided baseline performance of the memory task.

In the enumeration-alone condition, participants performed only the enumeration task. They were told to ignore items in initial and memory test displays. This provided enumeration performance without memory load as a baseline.

Participants completed eight blocks, with 30 trials per block, for each of the dual-task and the enumeration-alone conditions and one block of 30 trials for the memory-alone condition. The order of these blocks was counterbalanced across participants.

\section{Results}

Enumeration performance Fig. 2 shows performance on the enumeration task. Mean correct RTs (panel a) and errors (panel b) for enumeration responses are presented as a function of the number of items for both the dual-task condition and the enumeration-alone conditions.

For the dual-task condition, only trials with correct responses on both memory task and enumeration tasks were included in the RT analysis. In addition, numerosity data from trials with eight items were excluded from the following analyses, to avoid the end effect (e.g., Trick, 2005; Trick \& Pylyshyn, 1993; Watson et al., 2005). RTs greater than 2.5 standard deviations from the mean were excluded from further analyses (3.2\% of correct trials). Correct RTs in the enumeration task were subjected to a two-factor 2 (task condition: dual-task and enumeration- 
Fig. 2 Reaction times (a) and error rates (b) in enumeration task for enumeration-alone and dual-task condition in Experiment 1A. The error bars represent the standard errors of the means

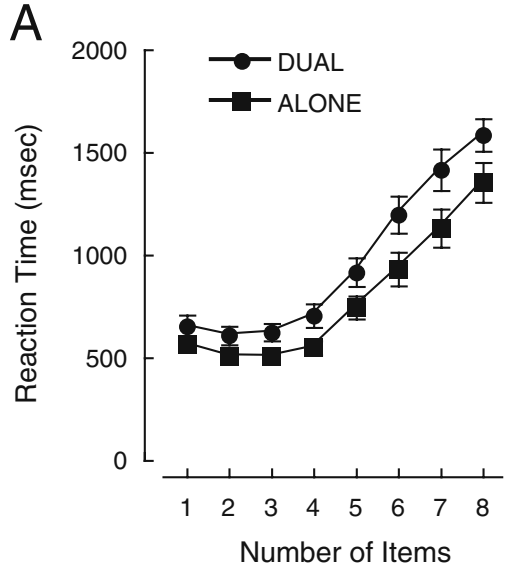

B

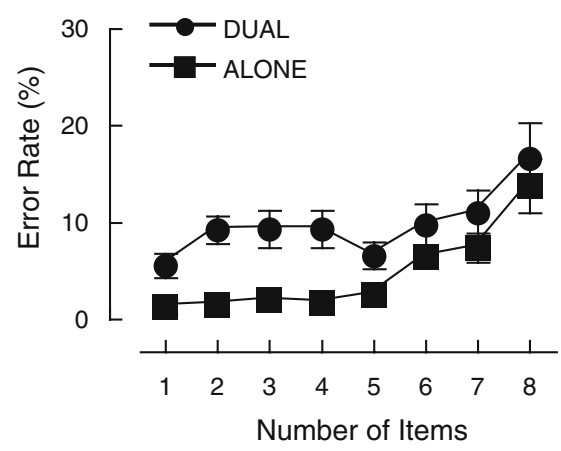

alone task) $\times 7$ (numerosity: one to seven) repeated measures ANOVA. The analysis revealed significant main effects of condition, $F(1,23)=26.62, p<.001$, and numerosity, $F(6,138)=86.76, p<.001$, and a significant interaction between them, $F(6,188)=6.921, p<.001$. RTs were shorter, overall, in the enumeration-alone condition than in the dual-task condition. A multiple comparison (Ryan's method) on the main effect of numerosity revealed that there were no differences among numerosities within four items, but all other numerosity comparisons showed significant differences $(p s<.05)$. The main effect of task condition was found at every point of numerosity. Although a significant interaction was found, no additional effects of numerosity were found in either of the two task conditions. To pursue this interaction, slopes of the RT functions as a function of number of items were calculated using the method of least squares.

Slopes were estimated separately for the subitizing range (one to three) and the counting range (five to seven) in each task condition; these appear in Table 1. Data from trials with four items were not included in the calculation, because the numerosity of four has been considered the boundary between subitizing and counting in previous studies (e.g., Trick \& Pylyshyn, 1993; Watson \& Maylor, 2006; Watson et al., 2007; Watson et al., 2005). Paired $t$-tests revealed a significant difference in slope between the dual-task and the enumeration-alone conditions in the counting range, $t(23)=$ $1.86, p<.05$, but not in the subitizing range, $t(23)<1$, n.s.

Subitizing range was determined by fitting a bilinear model to mean correct RTs for numerosities one to seven (Watson et al., 2005). This fitting yielded the slope change point of two linear functions for each participant. One participant was excluded from this analysis because the fitting failed to converge with reliable parameters. For each task condition, the estimated slope change points were averaged across participants, yielding the subitizing range. The subitizing ranges are shown in Table 2. The subitizing range was not different between these conditions, $t(22)<1$, n.s.

An ANOVA was performed on the error rate transformed by means of an arcsine transformation with the same two factors as the analysis for RTs. This analysis revealed significant main effects of numerosity, $F(1,23)=36.38, p<$ .001 , and condition, $F(6,138)=6.78, p<.001$, and a significant interaction, $F(6,138)=3.77, p<.005$. A multiple comparison showed significant differences between conditions in the range of one to five items. The effects of numerosity were due to the differences between the largest two numerosities (i.e., six and seven) and the other smaller numerosities (i.e., one to five). This effect was more apparent in the enumeration-alone condition than in

Table 1 Subitizing and counting slopes for enumeration-alone and dual-task conditions in Experiments 1A, 1B, 2A, and 2B

\begin{tabular}{|c|c|c|c|c|c|c|c|c|c|}
\hline \multirow[b]{2}{*}{ Condition } & \multirow[b]{2}{*}{ Load } & \multicolumn{2}{|c|}{ Experiment $1 \mathrm{~A}$} & \multicolumn{2}{|c|}{ Experiment 1B } & \multicolumn{2}{|c|}{ Experiment $2 \mathrm{~A}$} & \multicolumn{2}{|c|}{ Experiment 2B } \\
\hline & & Subitizing & Counting & Subitizing & Counting & Subitizing & Counting & Subitizing & Counting \\
\hline Enumeration alone & None & -30.7 & 187.9 & 21.2 & 309.1 & 5.3 & 261.8 & -12.8 & 237.0 \\
\hline \multirow[t]{3}{*}{ Dual task } & Low & -14.5 & 249.5 & 29.6 & 470.6 & - & - & 59.1 & 268.5 \\
\hline & Middle & - & - & 16.7 & 430.4 & 11.2 & 264.2 & 58.8 & 243.9 \\
\hline & High & - & - & 47.0 & 434.7 & - & - & 18.8 & 265.7 \\
\hline
\end{tabular}

The task conditions were also presented as load conditions corresponding to the number of to-be-remembered memory items imposed. Low-, middle-, and high-load corresponded with two, four, and six memory items, respectively. Subitizing and counting slopes were calculated from enumeration reaction times for $1-3$ and $5-7$, respectively 
Table 2 Subitizing ranges for enumeration-alone and dual-task conditions in Experiments $1 \mathrm{~A}, 1 \mathrm{~B}, 2 \mathrm{~A}$, and 2B

\begin{tabular}{llllll}
\hline Condition & Load & Experiment 1A & Experiment 1B & Experiment 2A & Experiment 2B \\
\hline Enumeration alone & None & 3.85 & 4.37 & 3.62 & 3.81 \\
Dual task & Low & 3.82 & 4.64 & - & 4.12 \\
& Middle & - & 4.41 & 4.08 & 3.89 \\
& High & - & 4.48 & - & 4.06 \\
\hline
\end{tabular}

The task conditions were also presented as the load conditions corresponding to the number of memory items imposed, in which low, middle, and highload corresponded to two, four, and six memory items, respectively

the dual-task condition. The interaction also shows that the effect of task condition was significant at each of less than six items.

Memory performance Fig. 3 depicts accuracy in the memory task as a function of numerosity (zero to seven) in the dual-task condition; also shown is performance in the memory-alone control condition (note that this corresponds to the zero number-of-items level). A one-way ANOVA with numerosity as a main factor yielded a significant main effect, $F(7,161)=3.09, p<.005$. A multiple comparison revealed that there was no interference in memory accuracy from concurrent enumeration for up to five items but that significant differences were found for six items relative to no and two items.

\section{Discussion}

Experiment 1A examined whether spatial working memory load interfered with enumeration processes. RTs indicated that this type of memory load had an effect only on enumeration in the counting range. Importantly, it affected neither subitizing efficiency nor subitizing range. If subitizing shared processes with spatial working memory, then, in

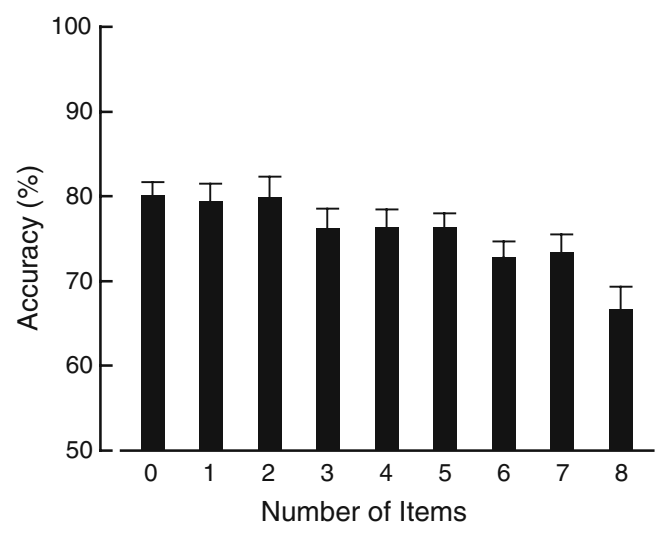

Fig. 3 Accuracy of spatial working memory task in Experiment $1 \mathrm{~A}$ as a function of the number of presented items in the intervening enumeration task, in which zero represents the memory-alone condition. The error bars represent the standard errors of the means the dual-task condition, we should have observed a decrease of the subitizing range and/or a decrease of enumeration efficiency (indexed by the slope) within the subitizing range. We observed neither. These results show that subitizing is functionally independent of spatial working memory capacity.

In the counting range, memory load selectively reduced enumeration efficiency as indexed by slope. This suggests that spatial working memory is involved in "counting." In order to enumerate more than about four items, the locations of items should be maintained in spatial working memory. Indeed, accuracy of the secondary memory task did not decrease within the subitizing range, but it did decrease with six items. It is possible that maintaining memory items competes with enumerating items for spatial working memory capacity in the counting range. This also supports the claim that counting requires some maintenance of spatial locations of visual items, using spatial working memory.

Although both enumeration efficiency and memory accuracy support the notion that spatial working memory has no role in subitizing, the results for enumeration accuracy painted a different picture. More errors of enumeration occurred in the dual-task condition than in the single-task condition, especially within the subitizing range. One may argue that this is evidence for the involvement of a spatial memory component in subitizing. However, there is another possibility, that this result may not be specific to spatial working memory but, instead, may be due to interference with the postsubitizing process that arose from the dual-task situations. We examined this possibility in Experiment $1 \mathrm{C}$.

RTs on the enumeration task were relatively longer in the dual-task condition than in the enumeration-alone condition, showing that the enumeration process overall was impeded by spatial working memory load. We propose that this cost reflects general interference levied by the secondary task. Although the overall increase in RT was not considered, in previous studies, to be a direct effect of interference (e.g., Woodman \& Luck, 2004; Woodman, Vogel, \& Luck, 2001), it may still be argued that the longer RTs observed in the present task reflect a general 
interference effect on enumeration due to working memory load.

Accordingly, in Experiment 1B, we further investigated whether enumeration RT varies with load in working memory. In this experiment, the dual-task condition presents memory displays containing two, four, or six tobe-remembered spatial positions. If memory load interferes with enumeration, we would expect to find that RT increases with the amount of the load to a point where this load exceeds the capacity of working memory (Emrich, AlAidroos, Pratt, \& Ferber, 2010). Given that working memory capacity has been estimated to be around four items, this implies that RT should be elevated as the number of to-be-remembered item positions increases from two to four items, but not for increases from four to six items. On the other hand, if enumeration is not affected by working memory load, RT should remain constant regardless of the amount of the load.

\section{Experiment 1B}

\section{Method}

The stimuli, apparatus, design, and procedure were identical to those in Experiment 1A, except for the following changes. In dual-task and memory-alone conditions, the number of positions to be remembered in the memory task (i.e., memory load) was varied: Two, four, or six positions of items could occur in a memory display. Instead of the serial presentation used in Experiment 1A, all memory items were presented simultaneously for $1,000 \mathrm{~ms}$. In the enumeration-alone condition, although two, four, or six items were randomly presented as memory items, as in Experiment 1A, participants were told to ignore these items. Each condition was performed in separate blocks with 16 trials per numerosity in each condition. Eighteen undergraduate and graduate students $(9$ males and 9 females $), 19-27$ years of age $(M=22.4$ years $)$ participated for payment. All reported normal or corrected-to-normal vision.

\section{Results and discussion}

The enumeration-alone condition was described as the nomemory load condition, and the dual-task condition was referred as low-, middle-, and high-load conditions according to the amount of the load, which were represented as continuous change of the memory load.

Enumeration performance Mean RTs in each memory load condition appear in Fig. 4. RT outliers were excluded from further analyses $(2.5 \%$ of correct trials). The

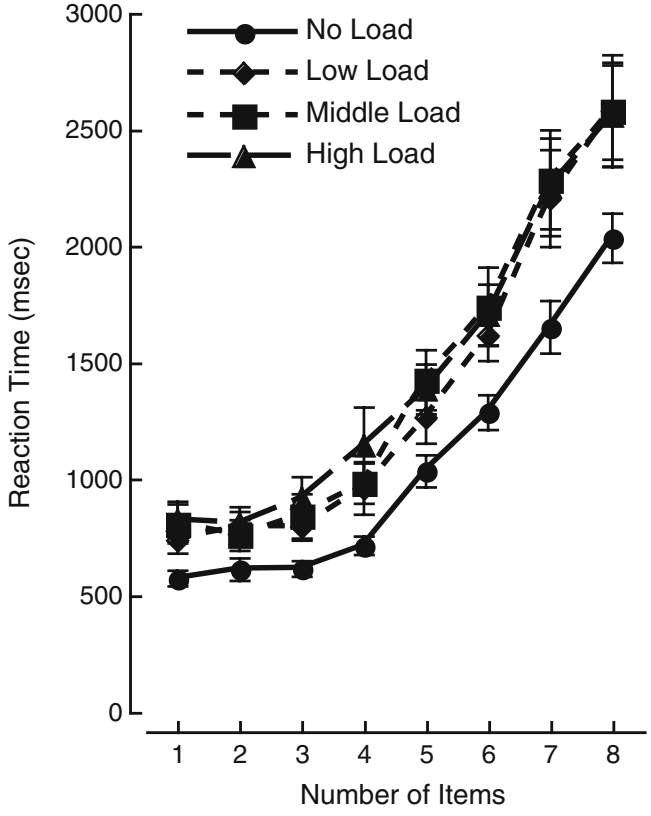

Fig. 4 Reaction times in enumeration task for each load condition in Experiment 1B. The error bars represent the standard errors of the means

procedures for all analyses were identical to those in Experiment 1A. Correct RTs were subjected to a two-way ANOVA with memory load (no-, low-, middle-, or highload condition) and numerosity (one to seven) as main factors. This analysis revealed main effects of memory load, $F(3,51)=12$. 79, $p<.001$, and numerosity, $F(6,102)=$ 101.16, $p<.001$, and an interaction, $F(18,306)=2.14, p<$ .005. A multiple comparison among load conditions showed that there was no difference among high-, middle-, and lowload conditions, but all three load conditions were significantly different from the no-load condition. For comparison among the numerosities, all comparisons show significant differences for more than four items. For the numbers of items smaller than five, RTs were significantly longer for four items than for one and two items, but the other comparisons were not significant. The interaction analysis was consistent with those of the main effects, in which no significant difference was found among low-, middle-, and high-load conditions at all numerosities. Furthermore, in all load conditions, significant differences were observed for the numerosities of more than four, but not less than five, except for the high-load condition, in which four showed significant differences from the smallest two numerosities, but not from five.

Subitizing and counting slopes for each load condition appear in Table 1. Separate ANOVAs for each range showed that the main effect of memory load was not significant in the subitizing range, $F(3,51)<1$. n.s., and was marginally significant in the counting range, $F(3,51)=$ 2.52, $p=.068$. The subitizing ranges, shown in Table 2, 
were estimated using the same procedure as in Experiment 1A. Data of 1 participant were excluded from the calculation due to poor fits. There was no significant main effect of memory load on the subitizing range, $F(3,48)<1$, n.s. Next, we examined the correlation between an individual's subitizing range in the enumeration-alone condition and his or her working memory capacity (as calculated by Cowan's (2001) $k$ formula) in the memory-alone condition. In this analysis, the peak $k$ value across different set sizes in each participant was adopted as that individual's working memory capacity. Results indicated that the $k$ values did not reliably correlate with subitizing range, $r=.24, p=.34$.

Error rates are shown in Table 3. There was a significant main effect of load condition, $F(3,51)=12.89, p<.001$, and of the interaction, $F(18,306)=1.80, p<.05$, but not of numerosity, $F(6,102)<1$. n.s. A multiple comparison revealed that error rates were significantly lower in the noload condition than in the other three load conditions, while no significant difference was found among the load conditions. Similar effects were observed in the interaction, except that there were more errors in the low-load condition than in the high-load condition at the numerosity of seven and more errors in the middle-load condition than in the noload condition at the numerosity of two.

Memory performance A two-way repeated measures ANOVA was conducted with the three memory load conditions (low, middle, and high) and numerosity (zero to seven) as main factors. The memory-alone condition was taken as a numerosity of zero for each load condition. This analysis revealed main effects of load, $F(2,34)=16.36, p<$ .001 , and numerosity, $F(7,119)=9.44, p<.001$, with no significant interaction, $F(14,238)<1$, n.s. A multiple comparison for the load conditions revealed that memory performance in the low-load condition was better than that in the two higher load conditions. This result showed that memory accuracy varied depending on the available capacity. The main effects of numerosity showed that errors were more frequent in numerosities of five and seven, as compared with zero to four, and in the numerosity of six, as compared with zero and one. These results suggested that memory for some positions did not interfere with enumeration for up to four items.
Experiment 1B replicated the main findings in Experiment $1 \mathrm{~A}$ and provided further evidence showing that subitizing was performed with a different capacity from working memory. If the increase of overall RT in the dual-task condition, relative to the enumeration-alone condition, is dependent on how much of the limited working memory capacity was occupied in Experiment 1A, we would expect that RTs should vary as a function of memory load in Experiment 1B. However, the result indicated that enumeration RTs were constant with the amount of the memory load. The subitizing slope and range also showed no change associated with memory load.

\section{Experiment 1C}

In Experiment 1A, enumeration errors were more frequent in the dual-task condition than in the enumeration-alone condition. One may argue that the increase of errors in the former was due to the concurrent spatial memory load. Experiment $1 \mathrm{C}$ addressed this interpretation. In the dual-task condition of Experiment 1A, participants reported the number of enumerated items; however, this report was always preceded by a memory matching report. In discussing Experiment 1A, we raised the possibility that this procedure may have caused interference with the postsubitizing process evident in dualtask situations, which then resulted in increased enumeration errors. To test this possibility, we examined whether the increase of errors in the dual-task condition would be replicated when the procedure was modified to minimize the requirements for an enumeration response.

In this experiment, participants reported the number of enumerated items before they reported memory matching. If enumeration errors are due to the concurrent spatial memory load, then, in Experiment 1C, we should observe the same pattern of results with respect to enumeration errors as that observed in Experiment 1A, despite these procedural changes.

\section{Method}

The stimuli, apparatus, design, and procedure were identical to those in Experiment 1A, except for the following. In
Table 3 Error rates (percentages) of enumeration task for enumeration-alone and dual-task conditions in Experiment 1B

The task conditions were also presented as the load conditions

\begin{tabular}{llllllllll}
\hline & \multicolumn{9}{c}{ Numerosity } \\
\cline { 3 - 9 } Condition & Load & 1 & 2 & 3 & 4 & 5 & 6 & 7 & 8 \\
\hline Enumeration alone & None & 0.6 & 1.3 & 0.3 & 0.6 & 1.3 & 1.7 & 2.0 & 4.5 \\
Dual task & Low & 7.5 & 4.2 & 7.1 & 8.2 & 8.4 & 6.5 & 13.0 & 13.7 \\
& Middle & 9.8 & 11.2 & 12.1 & 9.3 & 8.5 & 4.8 & 12.2 & 8.6 \\
& High & 7.9 & 6.9 & 11.3 & 10.4 & 6.3 & 8.9 & 5.0 & 4.6 \\
\hline
\end{tabular}


the dual-task and enumeration-alone conditions, the enumeration mask display was followed immediately by a beep, which was a cue for reporting the number of items. Thus, participants provided an enumeration report after a speeded response to the enumeration display. The retention interval was essentially $5,000 \mathrm{~ms}$; it was extended only if an enumeration response did not appear within the period. Twelve undergraduate or graduate students ( 3 males and 9 females), $18-25$ years of age ( $M=21.0$ years), participated for payment. All reported normal or corrected-to-normal vision.

Results and discussion

The results of Experiment 1C replicated those of Experiment 1A. RTs and error rates are shown in Fig. 5 for both the dualtask and the enumeration-alone conditions. As in the former experiments, enumeration RT increased with the number of items, $F(1,11)=7.46, p<.05$; moreover, overall RT was slightly shorter in the enumeration-alone condition than in the dual-task condition, $F(6,66)=2.41, p<.05$. Importantly, errors in the dual-task condition were comparable to those in the enumeration-alone condition; only the effect of numerosity was significant, $F(6,66)=4.67, p<.001$. The increase in enumeration errors found in Experiment $1 \mathrm{~A}$ was eliminated under the dual-task condition in Experiment 1C, where the number report preceded the memory report. No significant differences were observed in enumeration slopes between these conditions. Calculated subitizing ranges showed a small but significant difference between the dualtask condition and enumeration-alone condition (4.28 and 3.97 , respectively), $F(1,11)=5.05, p<.05$. The memory task also showed a result similar to that in Experiment 1A. As compared with the memory-alone condition, accuracy in the dual-task condition declined significantly when enumeration involved seven and eight items.

The rate for enumeration errors was comparable in the dual-task condition and the enumeration-alone condition when a numerical response was made immediately after a presentation of the enumeration display. This was inconsistent with the results in Experiments 1A and 1B, suggesting that the higher error rate in the dual-task conditions in these experiments derived from the fact that participants had to hold an enumerated number in mind while they retrieved, then reported, memory items. Therefore, the increase of enumeration errors in Experiment $1 \mathrm{~A}$ cannot be attributed to the load on visual working memory capacity.

The results of Experiment 1A, in which the rate for enumeration errors was higher in the subitizing range than in the counting range, suggested that the representation of an enumerated number suffered more severely from dual-task interference in subitizing than in counting. It is possible that a final enumeration number was not explicitly represented in the subitizing process because items were processed in a visual format. That is, visual items may have required additional processing to transform a visual format into a reportable verbal numerical representation prior to rendering a verbal response. In this case, such a transformation process is vulnerable to disruption by the other task, thereby leading to increased errors in Experiment 1A. This explanation is consistent with the notion that the nonzero subitizing slope reflected processes at the response stage that followed the subitizing process (Trick \& Pylyshyn, 1994). When no other task was required before numerosity responses were made, as in Experiment $1 \mathrm{C}$, the transforming process could not be disrupted. In addition, because counting may involve verbal one-by-one enumeration processes, a numerical representation is available for responses without additional processing for transformation. This implies that rendering a response in the memory task leads to interference only when it accompanies a report of the subitized, but not the counted, number. We supposed that the requirement to report remembered items prevents this transformational process; in turn, this will lead to a greater number of errors, as was found in Experiment 1A. In sum, the error increase in
Fig. 5 Reaction times (a) and error rates (b) in the enumeration task for enumeration-alone and dual-task conditions in Experiment 1C. The error bars represent the standard errors of the means

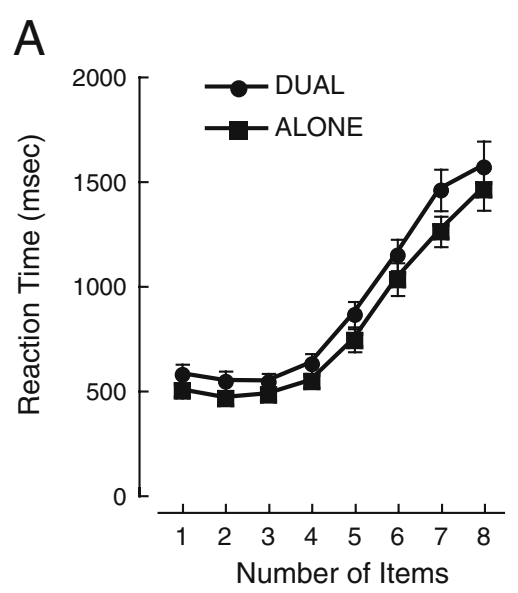

B

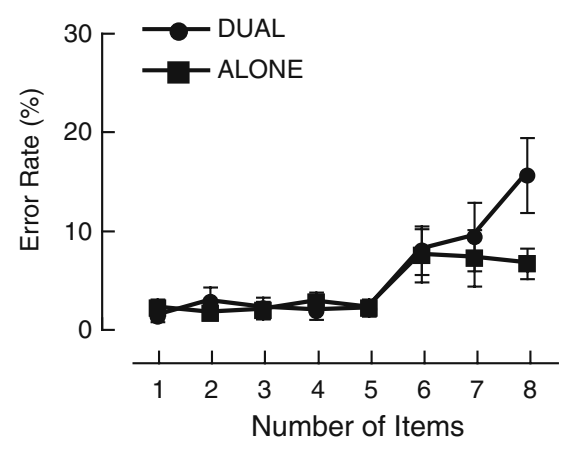


Experiment 1A does not necessarily reflect interference from the imposed load on working memory capacity.

\section{Experiment 2A}

In Experiment 2A, we explored the hypothesis that subitizing processes use nonspatial visual working memory, in which visual objects are represented as visual features, such as shape and color, regardless of their locations. If enumeration performance with a secondary task involving nonspatial visual working memory is the same as for that for enumeration when accompanied by a secondary task using spatial working memory (as in Experiment 1), this would indicate that subitizing operates independently of the particular nature of working memory-namely, whether it involves spatial or nonspatial subsystems.

\section{Method}

Participants Twelve undergraduate or graduate students ( 7 males and 5 females),19-27 years of age ( $M=21.5$ years), participated for payment. All had normal or corrected-tonormal vision.

Apparatus and stimuli The apparatus and stimuli were same as those in Experiment 1A, except for the memory items. A memory display comprised four gray shapes, shown in Fig. 1b. The memory items were randomly selected without replacement from a set of 26 novel shapes that were derived from a study on nonspatial visual working memory (Downing \& Dodds, 2004). The memory items were presented above, right of, below, and left of the central fixation cross, with a distance of $0.45^{\circ}$.

Design and procedure Experiment 2A included the same three task conditions as those in Experiment 1A: the enumeration-alone condition, the memory-alone condition, and the dual-task condition. Each trial consisted of the same sequence of displays in all three task conditions. The structure of the trials was identical to that in Experiment 1A, but the memory display contained a set of four items with novel shapes for 1,500 ms in this experiment, as shown in Fig. 1b.

In memory-alone and dual-task conditions, participants were required to indicate whether or not the initial and test memory displays were the same. On half the trials, the test display contained the same items at the same positions as the test display. In the remaining trials, only one item in the test display was replaced with a new item not presented in the initial display.

Each participant completed eight blocks with 32 trials per block for each of the dual-task and enumeration-alone conditions and one block of 32 trials for the memory-alone condition. The order of the blocks was counterbalanced across participants.

\section{Results}

Enumeration performance Fig. 6 shows mean correct RTs (panel a) and error rates (panel b) of the enumeration task as a function of number of items. All analyses were performed with statistical procedures identical to those used in Experiment 1A. RT outliers were excluded from further analysis $(3.0 \%$ of correct trials). Correct RTs and error rates for the enumeration task were subjected to a 2 (condition: dual-task and enumeration-alone) $\times 7$ (numerosity: one to seven) within-subjects ANOVA.

In the RT data, we found significant main effects of task condition, $F(1,11)=21.02, p<.001$, and numerosity, $F(6,66)=160.91, p<.001$, and a significant interaction between them, $F(6,66)=3.53, p<.005$. RTs were, overall, shorter in the enumeration-alone condition than in the dualtask condition. In addition, RTs increased with numerosity.
Fig. 6 Reaction times (a) and error rates $(\mathbf{b})$ in enumeration task for enumeration-alone and dual-task conditions in Experiment 2A. The error bars represent the standard errors of the means
A

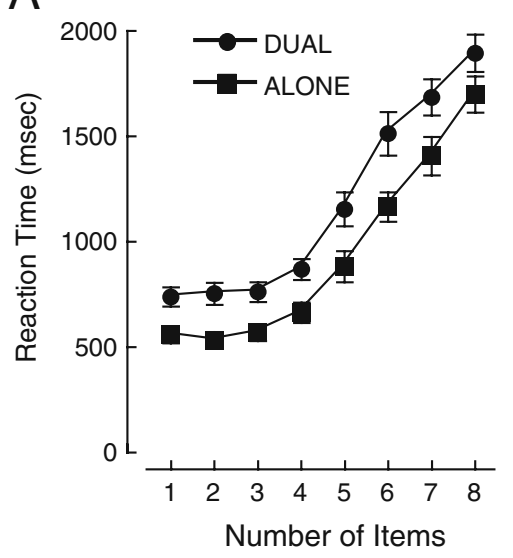

B

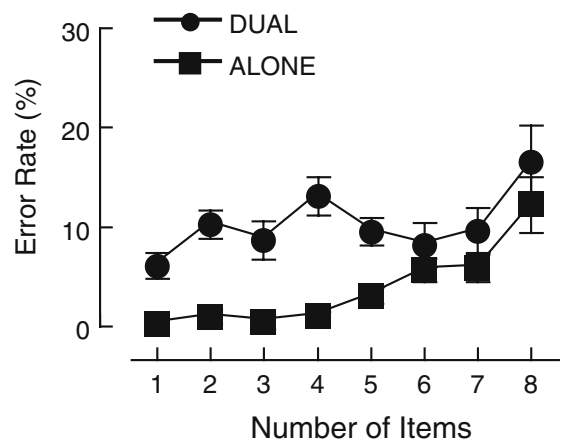


However, a multiple comparison revealed that RTs remained constant from one to three items and then increased from one or two to four items. All comparisons of RTs among other numerosities showed significant differences.

Enumeration slopes are shown in Table 1. A paired $t$-test revealed that there were no differences in the slopes between dual-task and enumeration-alone conditions, both in the counting range, $t(11)<1$, n.s., and in the subitizing range, $t(11)<1$, n.s. As shown in Fig. 5a, plotted lines of these two conditions are nearly parallel through the numerosities. This indicates that efficiency of enumeration was not affected, when participants performed the memory task, through the numerosities presented. Subitizing ranges are shown in Table 2 . There was a marginally significant difference, $t(11)=1.95, p=.076$.

An analysis of error rates revealed significant main effects of condition, $F(1,11)=12.04, p<.01$, and numerosity, $F(6,66)=3.71, p<.005$, and a significant interaction, $F(6,66)=3.33, p<.01$. More errors were made in the dual-task condition than in the enumeration-alone condition. Errors occurred more frequently at smaller numbers than at larger numbers. The interaction indicated that the effect of the condition was more profound in the range of one to five items than in larger numerosities. In the enumeration-alone condition, the effect of numerosity was found at six and seven items, relative to each of less than five items. In the dual-task condition, no significant effect of numerosity was found.

Memory performance Fig. 7 shows accuracy of memory tasks as a function of numerosity (zero to seven) in the dualtask condition. A one-way ANOVA with numerosity as a

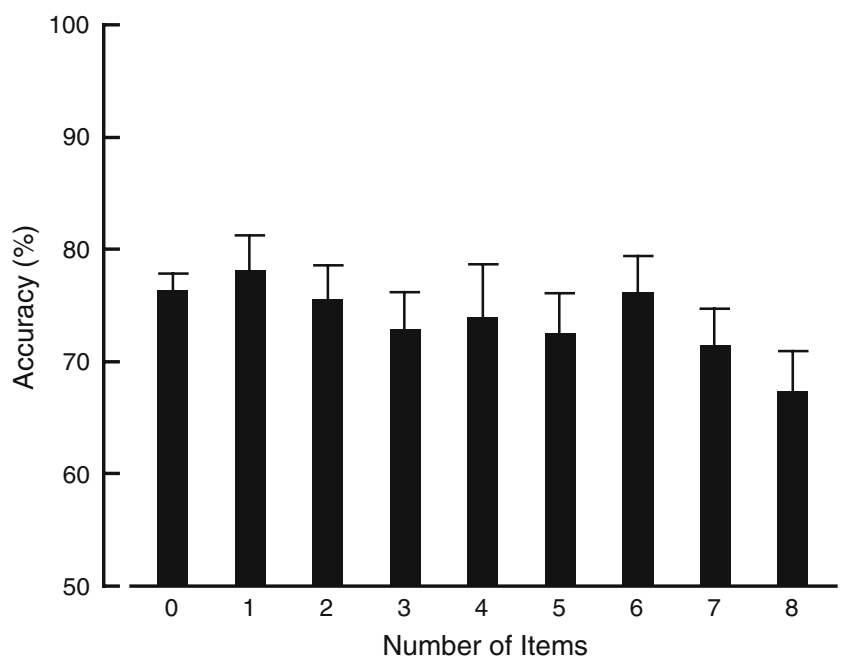

Fig. 7 Accuracy of nonspatial visual working memory task in Experiment $2 \mathrm{~A}$ as a function of the number of displayed items in the intervening enumeration task, in which zero represented the memory-alone condition. The error bars represent the standard errors of the means main factor revealed no effect of numerosity, $F(7,77)<1$, n.s., indicating that there was no systematic decrease in performance of the memory task with the number of items to be enumerated.

\section{Discussion}

Experiment 2A examined whether or not a nonspatial visual working memory load disrupts enumeration processes. Despite the additional memory task in the dual-task condition, both subitizing range and enumeration efficiency were comparable in the dual-task condition and the enumeration-alone condition. Furthermore, efficiency was not affected by memory load in either subitizing or counting. These findings indicate that nonspatial working memory load affected neither subitizing nor counting. In other words, in contrast to spatial working memory in Experiments $1 \mathrm{~A}$ and $1 \mathrm{~B}$, nonspatial visual working memory did not impede enumeration processes, regardless of the number of enumerated items. In addition, accuracy in the working memory task did not vary systematically with numerosity.

Errors of enumeration were more frequent in the dual-task condition than in the enumeration-alone condition, and these differences were more pronounced in the subitizing range than in the counting range. This result is consistent with Experiment $1 \mathrm{~A}$, even when a different type of memory task was used. This was also found in Experiment $1 \mathrm{C}$; hence, we attributed the error increase to interference from the intervening memory task response. Comparable findings in Experiments $1 \mathrm{~A}$ and $1 \mathrm{C}$ argue for an explanation based on interference that stems from the memory response that is common to both the spatial and nonspatial working memory tasks.

Again, overall RTs were longer in the dual-task condition than in the enumeration-alone condition in Experiment 2A. Woodman et al. (2001) reported a similar result using a visual search task. In their study, visual search efficiency was not affected, but overall RTs were prolonged by a concurrent nonspatial visual working memory task. Woodman et al.'s interpretation was that the memory task led to a slowing of a process that either proceeded or followed the actual visual search. Nevertheless, we examined whether the RT increase could reflect memory-dependent processes involved in the enumeration process, using the same rationale as in Experiment 1B.

\section{Experiment 2B}

Method

The stimuli, apparatus, design, and procedure were all identical to those used in Experiment 2A, except that two, 
four, or six items were presented as memory items. These memory items were presented simultaneously for $1,500 \mathrm{~ms}$. Eighteen undergraduate and graduate students (15 males and 3 females) $20-23$ years of age ( $M=21.4$ years), participated for payment. All reported normal or correctedto-normal vision.

Results and discussion

As in Experiment 1B, the enumeration-alone condition was referred to as the no-load condition, and the dual-task condition assumed three load levels: low, middle, and high load, according to the number of memory items involved.

Enumeration performance Mean RTs in each memory load condition appear in Fig. 8. All analyses were performed following the same statistical procedure as that used in Experiment 1B. RT outliers were excluded from further analysis ( $1.8 \%$ of correct trials). A two-way repeated measures ANOVA with memory load (no-, low-, middle-, and high-load conditions) and numerosity (one to seven) as main factors was conducted on correct RTs. There were main effects of memory load, $F(3,51)=16.06, p<.001$, numerosity, $F(6,102)=115.03$, and their interaction, $F(18,306)=1.72, p<.05$. Multiple comparisons showed that RTs were shorter in the no-load condition than in the other three load conditions, but importantly, there were no significant differences among the three memory load conditions. Effects of numerosity were due to slower

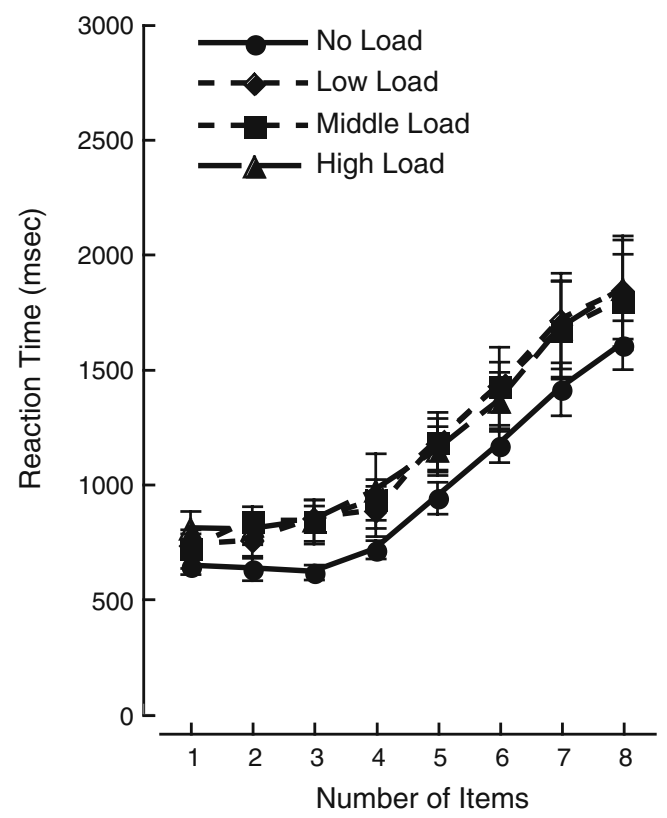

Fig. 8 Reaction times in the enumeration task for each load condition in Experiment 2B. The error bars represent the standard errors of the means responses to displays containing more than three items; significant RT increases were found at a numerosity of four, ascompared with one, but no other comparisons reached significance within one to four items. For the larger numerosities, RTs increased with the number of items. The interaction of memory load with numerosity indicated that effects of memory load were attenuated with numerosity. The no-load condition was different from the lowload condition when enumeration displays contained more than two items, and it was different from the middle-load conditions when enumeration displays contained more than one item; finally, the no-load condition was significantly different from high-load condition enumeration displays at all numerosity levels. However, comparisons among these three load conditions showed no differences at any of the numerosity levels of enumeration displays. The effects of numerosity were similar in all the load conditions, in which significant differences were not found within four items, and then, from five items, RTs increased with the number of enumeration items.

Enumeration error rates are shown in Table 4. A twoway repeated measures ANOVA of error rates revealed a significant effect of load, $F(3,51)=12.88, p<.001$, a marginally significant effect of numerosity, $F(6,102)=$ 2.185, $p=.051$, and a significant interaction, $F(18,306)=$ $1.72, p<.05$. Enumeration was more accurate in the noload condition than in the other three load conditions. The interaction indicated that performance in the no-load condition was more accurate than in the other memory load conditions at the numerosities of one, two, four, and seven.

Subitizing and counting slopes for each load condition are shown in Table 1. Separate ANOVAs were conducted for each slope, with the memory load as a main factor. A main effect was found only for the subitizing slope, $F(3,51)=$ $2.98, p<.05$. However a multiple comparison follow-up of this main effect failed to identify any significant differences among the four means. No significant difference among the four memory load conditions was found in counting slope, $F(3,51)<1$. n.s. Subitizing ranges were also calculated from RT data, except for 2 participants who did not provide a converging fitting result. As is shown in Table 2, there was no significant effect of memory load, $F(3,45)<1$, n.s. Correlation analyses involving individual subitizing ranges showed no evidence of a reliable association of range with individual $k$-estimates, $r=.13, p=.61$.

Memory performance A two-way repeated measures ANOVA was conducted for memory accuracy with memory load and numerosity (zero to seven) as main factors. This analysis revealed main effects of memory load, $F(2,34)=$ $124.92, p<.001$, and numerosity, $F(7,119)=12.33, p<.001$, but no significant interaction, $F(14,238)=1.35$, n.s. All 
Table 4 Error rates (percentages) of enumeration task for enumeration-alone and dual-task conditions in Experiment 2B

task conditions were also presented as the load conditions

\begin{tabular}{llllllllll}
\hline \multicolumn{1}{c}{ Numerosity } \\
\cline { 3 - 9 } Condition & Load & 1 & 2 & 3 & 4 & 5 & 6 & 7 & 8 \\
\hline Enumeration alone & None & 1.3 & 1.0 & 1.0 & 1.0 & 2.0 & 4.5 & 3.1 & 13.5 \\
Dual task & Low & 12.9 & 5.1 & 7.5 & 8.3 & 4.2 & 7.8 & 7.0 & 13.6 \\
& Middle & 12.4 & 8.7 & 8.0 & 8.7 & 4.5 & 8.0 & 16.5 & 12.4 \\
& High & 5.2 & 8.9 & 3.2 & 10.2 & 7.6 & 7.5 & 7.1 & 12.1 \\
\hline
\end{tabular}

comparisons among the three load conditions showed significant differences, with accuracy decreasing with increases in memory load. Enumeration numerosity also affected memory performance, but no systematical changes were found as a function of numerosity.

Experiment $2 \mathrm{~B}$ provides a result consistent with that in Experiment 2A: It demonstrates that enumeration efficiency does not vary as a function of the concurrent nonspatial memory load. The result indicates that the overall increase in RT arises from general memory load irrespective of the number of items to be remembered.

\section{General discussion}

The present study used a dual-task procedure based on combining a primary enumeration task with two different types of secondary tasks, involving visual working memory. The main goal was to examine whether enumeration performance-in particular, the range and efficiency of subitizing - was affected by the available capacity of working memory. In Experiments $1 \mathrm{~A}$ and $1 \mathrm{~B}$, participants were asked to perform an enumeration task while carrying out a spatial working memory task in which they were required to remember several locations of visual objects. To perform the enumeration task successfully, it was assumed that the locations of objects that have already been enumerated must be remembered in order to avoid enumerating these items twice. The rationale is that subitizing is based on the available capacity of spatial working memory. If so, both the range and efficiency of subitizing should be attenuated by the presence of spatial working memory load in the dual-task condition. However, in Experiment 1A, although counting efficiency of displays with larger numbers of objects was affected, we did not find that the concurrent spatial working memory load reduced either the range or the efficiency of subitizing. Experiment $1 \mathrm{~B}$ also showed that RTs were not affected by variations in concurrent spatial memory load. Finally, Experiment 1C provided evidence that the increase of errors under the dualtask condition in Experiment 1A was not associated with the spatial working memory load.
Experiments 2A and 2B examined dual-task effects when the secondary task involved nonspatial visual working memory. Participants were required to remember several novel shapes of objects while they enumerated other targets. Experiment 2A showed that memory load failed to reduce either the range or the efficiency of subitizing; in fact, it also failed to affect counting efficiency. Furthermore, Experiment 2B showed that RTs remained constant over different levels of nonspatial memory load. Moreover, there was no correlation of individuals' subitizing ranges with estimated capacity of either spatial or nonspatial working memory. These results suggest that spatial and nonspatial working memory are not processes that explain the profile of enumeration performance over a range of different numerosities.

Together, these experiments reveal that the subitizing range is not determined by the capacity of visual working memory, whether it involves spatial or nonspatial working memory. These results suggest that a similar capacity between subitizing and working memory does not reflect a common capacity limitation. Although previous studies have shown that enumeration appears to be unaffected by disruption of a memory rehearsal process with either a secondary verbal working memory task (Logie \& Baddeley, 1987) or a secondary tapping task (Trick, 2005), these studies did not manipulate visual working memory capacity under an enumeration task. The present research provides direct evidence that the capacity of spatial and visual working memory is not associated with subitizing. In short, it appears that subitizing is free from any working memory capacity limitations.

This dissociation of subitizing capacity from visual working memory capacity may be due to different processing stages that have different limitations. $\mathrm{Xu}$ and Chun (2009) suggested that the visual system employs two sequential stages, which involve an object individuation process and an object identification process, respectively. During the object individuation process, about four objects are individuated or selected solely via their spatial position. In the second, object identification stage, feature information of the selected objects is putatively represented, in which the number of the objects varies depending on the object's complexity. According to this account, subitizing reflects the capacity limit of the object individuation stage 
and visual working memory reflects the capacity limit of the object identification stage. This account is also consistent with the present results, in that we found no correlation between individual subitizing range and visual working memory capacity. Further research is required to address this possibility.

This raises the question of how subitizing is accomplished, if it is not determined by limited capacities related to visual working memory. Because visual working memory was not engaged, our results and previous findings suggest that constraints that result in subitizing are confined to processes that precede both attention and working memory. We suggest that enumeration operates via a master locationmap that represents locations, not features, of objects (Watson \& Maylor, 2006). Watson and Maylor reported that enumeration for items with two different colors was comparable to enumeration of those items with identical colors, suggesting that subitizing operated in a feature-independent fashion, at least with respect to items defined by color. Such results alone cannot exclude the possibility that limits on subitizing are somehow due to working memory. Nevertheless, they dovetail with the present results in suggesting that subitizing occurred on a location map, which was located earlier than working memory representations in terms of the functional architecture of visual processing (Wolfe, 1994).

The hypothesis that a location map is involved in subitizing is consistent with the FINST account. That is, Pylyshyn and Storm (1988) proposed that FINST referred to the limited number of locations on the representation of an intermediate processing between preattentive and attentive levels. Watson and Maylor (2006) have also suggested that FINST referred to location-based representation coding salience of objects in a visual display. However, in the end, this account remains unclear regarding the precise determinants of subitizing range. It is possible that this range reflects the capacity of a spatial reference mechanism, one that resembles the number of mental tokens postulated for FINST and implemented in the visual system.

Although the manipulation of spatial working memory had little impact on subitizing, Experiment 1A revealed that it did have an impact on counting. Counting was less efficient with spatial working memory load than without this load. This suggests that interference arises from a simultaneous working memory task and that representations of spatial locations stored in spatial working memory are used for counting. Counting might be mediated with spatial working memory in which the locations of enumerated objects are tagged. The fact that spatial working memory load differentially affected subitizing and counting is inconsistent with previous studies proposing the operation of a unified enumeration process that accounts for both subitizing and counting. For example, Watson, Maylor,
Allen, and Bruce (2007) argued that FINST is applied for both subitizing and counting. Especially, with respect to remembering the locations of objects already enumerated (i.e., tagging), Watson et al. (2007) suggested that the tagging of items was preformed with FINST regardless of the number of items. However, the present result suggests another possibility, that the counting required spatial working memory capacity in order to keep tags of the already enumerated items, but not in subitizing. In other words, the present study was in favor of partially different mechanisms for subitizing and counting. Apparently, however, further works are required to elucidate the issue.

In summary, the present study provides two primary findings. First, subitizing is not mediated by a limited capacity specific to either spatial or nonspatial working memory. Second, counting requires spatial working memory. Together, these two findings lead to the conclusion that subitizing reflects the operation of a limited capacity mechanism that is not associated with either spatial or nonspatial working memory. However, it remains unclear how such a rapid enumeration for subitizing could be achieved without involving some kind of working memory system. At a minimum, we revealed that subitizing is a unique process independent of known memory processes. Therefore, further studies are required to reveal why the range is limited at approximately four items.

\section{References}

Baddeley, A. D., \& Logie, R. H. (1999). Working memory: The multiple component model. In P. Shah \& A. Miyake (Eds.), Models of working memory (pp. 28-61). Cambridge: Cambridge University Press.

Barrouillet, P., Lépine, R., \& Camos, V. (2008). Is the influence of working memory capacity on high level cognition mediated by complexity or resource-dependent elementary processes? Psychonomic Bulletin \&Review, 15, 528-534.

Brainard, D. H. (1997). The psychophysics toolbox. Spatial Vision, $10,433-436$.

Cocchini, G., Logie, R. H., Della Sala, S., MacPherson, S. E., \& Baddeley, A. D. (2002). Concurrent performance of two memory tasks: Evidence for domain-specific working memory systems. Memory \& Cognition, 30, 1086-1095.

Cowan, N. (2001). The magical number 4 in short-term memory: A reconsideration of mental storage capacity. Behavioral and Brain Sciences, 24, 87-185.

Downing, P. E., \& Dodds, C. M. (2004). Competition in visual working memory for control of search. Visual Cognition, 11, 689-703.

Emrich, S. M., Al-Aidroos, N., Pratt, J., \& Ferber, S. (2010). Finding memory in search: The effect of visual working memory load on visual search. Quarterly Journal of Experimental Psychology, 63, 1457-1466.

Frick, R. (1987). The homogeneity effect in counting. Perception \& Psychophysics, 41, 8-16.

Jensen, E. M., Reese, E. P., \& Reese, T. W. (1950). The subitizing and counting of visually presented fields of dots. Journal of Psychology, 30, 363-392. 
Jevons, W. (1871). The power of numerical discrimination. Nature, 3, 281-282.

Kaufman, E., Load, M., Reese, T., \& Volkmann, J. (1949). The discrimination of visual number. American Journal of Psychology, 62, 498-525.

Klahr, D. (1973). A production system for counting, subitizing, and adding. In W. G. Chase (Ed.), Visual information processing (pp. 527-546). New York: Academic Press.

Logie, R. H. (1995). Visuo-spatial processing in working memory. Hove, U.K.: Erlbaum.

Logie, R. H., \& Baddeley, A. D. (1987). Cognitive processes in counting. Journal of Experimental Psychology: Learning, Memory, and Cognition, 13, 310-326.

Luck, S. J., \& Vogel, E. K. (1997). The capacity of visual working memory for features and conjunctions. Nature, 390, 279-281.

Mandler, G., \& Shebo, B. J. (1982). Subitizing: An analysis of its component processes. Journal of Experimental Psychology: General, 111, 1-22.

Oyama, T., Kikuchi, T., \& Ichihara, S. (1981). Span of attention, backward masking and reaction time. Perception \& Psychophysics, 29, 106-112.

Pashler, H. (1988). Familiarity and visual change detection. Perception \& Psychophysics, 44, 369-378.

Pelli, D. G. (1997). The VideoToolbox software for visual psychophysics: Transforming numbers into movies. Spatial Vision, 10, 437-442.

Pylyshyn, Z. (1989). The role of location indices in spatial perception: A sketch of the FINST spatial-index model. Cognition, 32, 6597.

Pylyshyn, Z., \& Storm, R. W. (1988). Tracking multiple independent targets: Evidence for a parallel tracking mechanism. Spatial Vision, 3, 179-197.

Trick, L. (2005). The role of working memory in spatial enumeration: Patterns of selective interference in subitizing and counting. Psychonomic Bulletin \& Review, 12, 675-681.
Trick, L., \& Pylyshyn, Z. (1993). What enumeration studies can show us about spatial attention: Evidence for limited capacity preattentive processing. Journal of Experimental Psychology: Human Perception and Performance, 19, 331-351.

Trick, L., \& Pylyshyn, Z. (1994). Why are small and large numbers enumerated differently? A limited-capacity preattentive stage in vision. Psychological Review, 101, 80-102.

Tuholski, S. W., Engle, R. W., \& Baylis, G. C. (2001). Individual differences in working memory capacity and enumeration. Memory \& Cognition, 29, 484-492.

Vogel, E. K., Woodman, G. F., \& Luck, S. J. (2001). Storage of features, conjunctions, and objects in visual working memory. Journal of Experimental Psychology: Human Perception and Performance, 27, 92-114.

Watson, D. G., \& Maylor, E. A. (2006). Effects of color heterogeneity on subitization. Perception \& Psychophysics, 68, 319-326.

Watson, D. G., Maylor, E. A., Allen, G., \& Bruce, L. A. M. (2007). Early visual tagging: Effects of target-distractor similarity and old age on search, subitization, and counting. Journal of Experimental Psychology: Human Perception and Performance, 33, 549-569.

Watson, D. G., Maylor, E. A., \& Bruce, L. A. M. (2005). The efficiency of feature-based subitization and counting. Journal of Experimental Psychology: Human Perception and Performance, $31,1449-1462$.

Wolfe, J. M. (1994). Guided Search 2.0: A revised model of visual search. Psychonomic Bulletin \& Review, 1, 202-238.

Woodman, G. F., \& Luck, S. J. (2004). Visual search is slowed when visuospatial working memory is occupied. Psychonomic Bulletin \& Review, 11, 269-274.

Woodman, G. F., Vogel, E. K., \& Luck, S. J. (2001). Visual search remains efficient when visual working memory is full. Psychological Science, 12, 219-274.

Xu, Y., \& Chun, M. M. (2009). Selecting and perceiving multiple visual objects. Trends in Cognitive Sciences, 13, 167-174. 